\title{
The laminar/turbulent transition for paste sheet flow
}

\author{
P.T. Slatter Rheology and Materials Processing Centre, RMIT University, Australia
}

R. Haldenwang Cape Peninsula University of Technology, South Africa

R.P. Chhabra Indian Institute of Technology, India

\begin{abstract}
The sheet flow of paste material is of critical importance in tailings disposal, since this is the principal flow mechanism at tailings disposal sites. Furthermore, the sheet flow paradigm for free surface flows has the potential to provide a basis for analysis and design for the open channel flow of mineral tailings suspensions. In our previous studies, we showed that a sheet flow diagram could be constructed, and that it is directly related to the rheogram in a manner analogous to that of Rabinowitsch and Mooney for tube flow. Furthermore we showed that these flows could be scaled for engineering design purposes in a manner similar to the approach of Metzner and Reed for tube flow. Whilst some progress has been made for the rheometrical measurement and analyses of sheet flow, many unresolved issues remain. Arguably, the most acute of these is the location of the Laminar/Turbulent transition. The objective of this paper is to develop and evaluate an approach for the analysis of the Laminar/Turbulent transition of sheet flows. Building on our previous studies, a new Reynolds number approach for sheet flows of general non-Newtonian material is developed and presented. Using experimental data, it is shown that this approach works well for power law fluids, and extension of this approach for the analysis of viscoplastic materials is discussed. Further work involving the role of the yield stress in the transitional flow process is being undertaken.
\end{abstract}

\section{Introduction}

The mining and mineral processing industry is under continuous pressure to save water, and tailings disposal is a potential area of significant water savings. With increase in concentration the tailings materials become more viscous and non-Newtonian in nature and the flow behaviour more difficult to predict. Ultimately viscous forces dominate and the flow becomes laminar. After exit from the spigot, the material will flow as an unconfined sheet at the disposal site. The rheological properties of this sheet flow will profoundly affect the operation of the disposal facility. The broad aim of this paper is to continue development of a new approach for the analysis of the laminar sheet flow of a general time independent non-Newtonian material. In particular, we will extend the use of a new scaling parameter developed from fundamental principles, which relates the sheet flow directly to its rheological properties. The main problem is the engineering design of free surface flows in open channels. For turbulent flow, the flow behaviour is similar to that of water, and much success has resulted from imposing the "pipe flow paradigm" (Slatter et al., 2010). However, for laminar flow, the flow behaviour is markedly dissimilar to that of water, and limited success has been achieved by imposing the pipe flow paradigm.

In seeking a way forward we note that there are only two flow situations where the shear stress distribution is accessible:

- cylindrical tube flow

- sheet flow (planar flow/slit flow).

Many rheometric geometries have been developed from these, and these two situations can be considered to form the extremes for this engineering design context. Most open channel (free surface) flow applications lie somewhere between these two and these intermediate flow situations have shear stress distributions which are not readily accessible.

As introduced above, (Chow, 1959; Fuentes, 2004; Haldenwang et al., 2002) the first attempt to deal with this has historically been to impose the pipe flow paradigm, using the "equivalent diameter" or hydraulic 
radius concept. This approach has some fundamental problems (Slatter et al., 2010) in laminar and transitional flows, the principal weaknesses being:

- the relationship between Reynolds number and friction factor for laminar flow is not constant, and varies with shape

- transition does not occur at some fixed value of Reynolds number

- a slope effect, which is effectively hidden by the log-log scale customarily used for presenting Moody type diagrams.

To remedy these fundamental problems, we propose to impose a sheet flow paradigm. This approach was used to extend our previously developed sheet flow analysis to general open channel flow analysis. Furthermore, we showed that such sheet flow analysis could be used in two ways:

- rheometrical analysis

- engineering design of open channel flow.

Having achieved some success in this direction, arguably the most important next step - and the objective of this present paper - is to develop and evaluate a criterion for reliably determining the laminar/turbulent transition for the sheet flow paradigm.

\section{Theory and literature}

The shear stress $\tau$ for sheet flow increases linearly with depth $h$, from zero at the free surface to the wall shear stress $\tau_{0}$ at depth $H$ as indicated in Figure 1. The shear stress distribution is:

$$
\tau=\rho g h \sin \alpha
$$

Where:

$\tau \quad=\quad$ the shear stress at depth $h(\mathrm{~Pa})$.

$\rho \quad=\quad$ the fluid density $\left(\mathrm{kg} / \mathrm{m}^{3}\right)$.

$g=$ gravitational acceleration $\left(\mathrm{m} / \mathrm{s}^{2}\right)$.

$h \quad=\quad$ the depth of fluid in the sheet $(\mathrm{m})$.

$\alpha=$ inclination (degrees).

The shear stress distribution is shown in Figure 1.

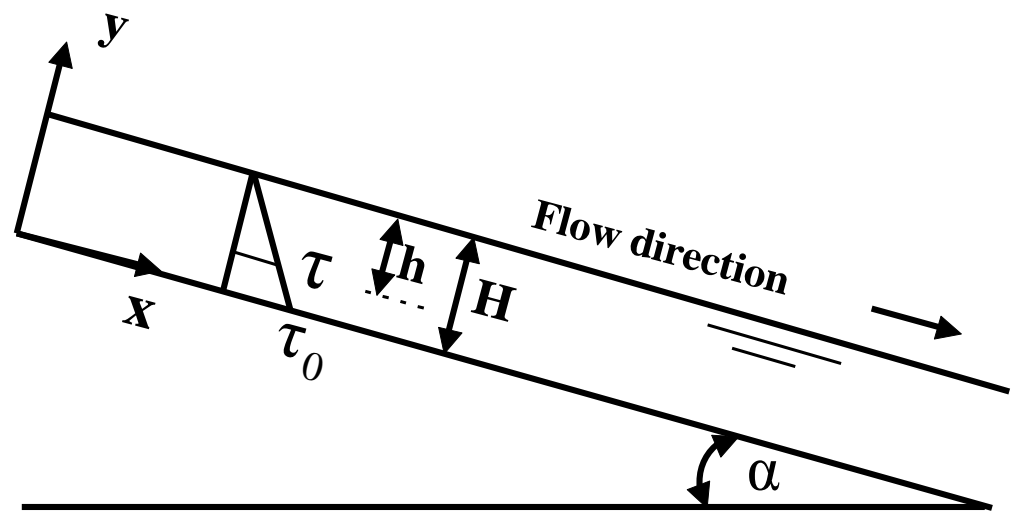

Figure 1 Shear stress distribution for sheet flow (Chow, 1959)

The equation for laminar Newtonian sheet flow is obtained by integrating Equation (1) over the flow depth (Chow, 1959): 


$$
Q=\frac{H^{2} \tau_{0}}{3 \mu}
$$

Where:

$$
\begin{array}{lll}
Q & = & \text { volumetric flow rate per unit channel width }\left(\mathrm{m}^{3} / \mathrm{s} / \mathrm{m}\right) . \\
H & = & \text { fluid height }(\mathrm{m}) . \\
\tau_{0} & = & \text { wall shear stress }(\mathrm{Pa}) . \\
\mu & = & \text { dynamic viscosity (Pa.s). }
\end{array}
$$

In terms of the mean velocity $V$ :

$$
V=\frac{Q}{H}=\frac{H \tau_{0}}{3 \mu}
$$

where:

$V \quad=\quad$ mean velocity $(\mathrm{m} / \mathrm{s})$.

For a Newtonian fluid the shear rheology relationship is:

$$
\not=\frac{\tau}{\mu} \text { and } \quad \& \quad=\frac{\tau_{0}}{\mu}
$$

where $\dot{\gamma}=$ the shear rate $\left(\mathrm{s}^{-1}\right)$ and $\dot{\gamma}_{0}=$ the wall shear rate $\left(\mathrm{s}^{-1}\right)$. If Equations (3) and (4) are combined and if this is expressed in terms of the wall shear stress and the wall shear rate the relationship is as follows:

$$
\tau_{0}=\mu \frac{3 V}{H}=\mu \varkappa \&
$$

therefore:

$$
\nLeftarrow=\frac{3 V}{H}
$$

which gives us the bulk sheet flow shear rate for sheet flow as $3 \mathrm{~V} / \mathrm{H}$.

The wall shear stress for sheet flow is:

$$
\tau_{0}=\rho g H \sin \alpha
$$

Laminar sheet flow can therefore be represented and analysed as a unique relationship between wall shear stress $\tau_{0}$ and bulk shear rate $3 \mathrm{~V} / \mathrm{H}$, as is customarily done for pipe flow.

The sheet flow analysis for a general time independent non-Newtonian fluid can be obtained (Slatter et al., 2010) in terms of the bulk shear rate as:

$$
\left.\left.\frac{3 V}{H}=\frac{3}{\tau_{0}^{2}} \int_{0}^{\tau_{0}} \tau\right\rangle \& \tau\right) d \tau
$$

By analogy with tube flow, one can introduce the apparent sheet flow behaviour index $n_{*}^{\prime}$ defined as the slope of the logarithmic plot of wall shear stress $\tau_{0}$ versus bulk shear rate $3 V / H$ (Slatter et al., 2010). Finally, the shear rate at the wall is expressed in terms of the bulk shear rate $(3 \mathrm{~V} / \mathrm{H})$ as (Slatter et al., 2010):

$$
\nLeftarrow=\frac{3 V}{H}\left(\frac{2 n_{*}^{\prime}+1}{3 n_{*}^{\prime}}\right)
$$

In summary we recapitulate in Table 1 the analogy of the proposed sheet flow analysis with that of Rabinowitsch and Mooney for tube flow. 
Table 1 Comparison of key elements of the rheometric analysis of tube to sheet flow (Slatter et al., 2010)

\begin{tabular}{lccc}
\hline & $\begin{array}{c}\text { Bulk Shear } \\
\text { Rate }\end{array}$ & $\begin{array}{c}\text { Wall Shear } \\
\text { Stress }\end{array}$ & $\begin{array}{c}\text { R-M } \\
\text { Factor }\end{array}$ \\
\hline Tube flow & $\frac{8 V}{D}$ & $\frac{D \Delta p}{4 L}$ & $\frac{3 n^{\prime}+1}{4 n^{\prime}}$ \\
Sheet flow & $\frac{3 V}{H}$ & $\rho g H \sin \alpha$ & $\frac{2 n_{*}^{\prime}+1}{3 n_{*}^{\prime}}$ \\
\hline
\end{tabular}

\section{$3 \quad$ New model}

The apparent sheet flow behaviour index $n_{*}^{\prime}$ has been defined in terms of a power law relationship between the wall shear stress $\tau_{0}$ and the bulk shear rate $3 V / H$. After the method of Metzner and Reed (1955), we can define an apparent sheet flow fluid consistency index $K_{*}^{\prime}$ as:

$$
K_{*}{ }^{\prime}=\frac{\tau_{0}}{\left(\frac{3 V}{H}\right)^{n_{*}}} .
$$

This leads to the fundamentally important laminar flow design equation for sheet flow as:

$$
\tau_{0}=K_{*}^{\prime}\left(\frac{3 V}{H}\right)^{n_{*}^{\prime}} .
$$

In order to accommodate the reality that actual channels may approach sheet flow, but will always have side edges at some point, we replace $H$ with the Hydraulic radius $R_{h}$ so that:

$$
\tau_{0}=K_{*}{ }^{\prime}\left(\frac{3 V}{R_{h}}\right)^{n_{*^{\prime}}} .
$$

We can now define a new Reynolds number for sheet flow after the Newtonian paradigm:

$$
\operatorname{Re}_{4}=\frac{8 \rho V^{2}}{\tau_{0}}
$$

Combing Equations (12) and (13) we have:

$$
\operatorname{Re}_{4}=\frac{8 \rho V^{2}}{K_{*}^{\prime}\left(\frac{3 V}{R_{h}}\right)^{n_{*}}} .
$$

It is expected that this Reynolds number will provide a basis for the establishment of dynamic similarity for sheet flow. In particular, this new Reynolds number should provide a basis for the discrimination between laminar and turbulent flow.

\section{$4 \quad$ Experimental procedure and results}

The experimental work was conducted by the Flow Process Research Centre at the Cape Peninsula University of Technology in a $10 \mathrm{~m}$ long tilting flume which is $300 \mathrm{~mm}$ wide with slopes from 1-5 degrees. The maximum flow rate achieved by the progressive cavity positive displacement pump is $24 \mathrm{~L} / \mathrm{s}$ but higher flow rates up to $45 \mathrm{~L} / \mathrm{s}$ were achieved using a centrifugal pump. The flow heights in the channel were 
measured using digital depth gauges that were linked to the computer but had to be lowered manually to the surface of the fluid in the flume.

The rheology of the fluids was measured in a tube viscometer with three tubes $(13,28$ and $80 \mathrm{~mm}$ diameter respectively) which is in-line with the tilting flume. Magnetic flow meters and differential pressure transducers are fitted to each line for measuring flow rates and pressure drops. A mass flow meter is fitted to the $13 \mathrm{~mm}$ line which can also provide density and temperature readings. More detail is provided with regard to the experimental procedures and accuracies obtained are presented in Haldenwang (2003) and Haldenwang and Slatter (2006).

In Figure 2 a plot of wall shear stress versus bulk shear rate for a 3.8\% carboxymethyl cellulose (CMC) solution is presented. Each slope is presented separately. It is important to note is that the laminar flow data is co-linear at the different channel slopes, as would customarily be seen for tube viscometer data plotted on a pseudo shear diagram, for different diameters. With tube viscometry data the transition and subsequent turbulent data can be observed. A similar phenomenon for the flume data is seen in Figure 2 - here the transition and subsequent turbulent data can be clearly observed for the different channel slopes.

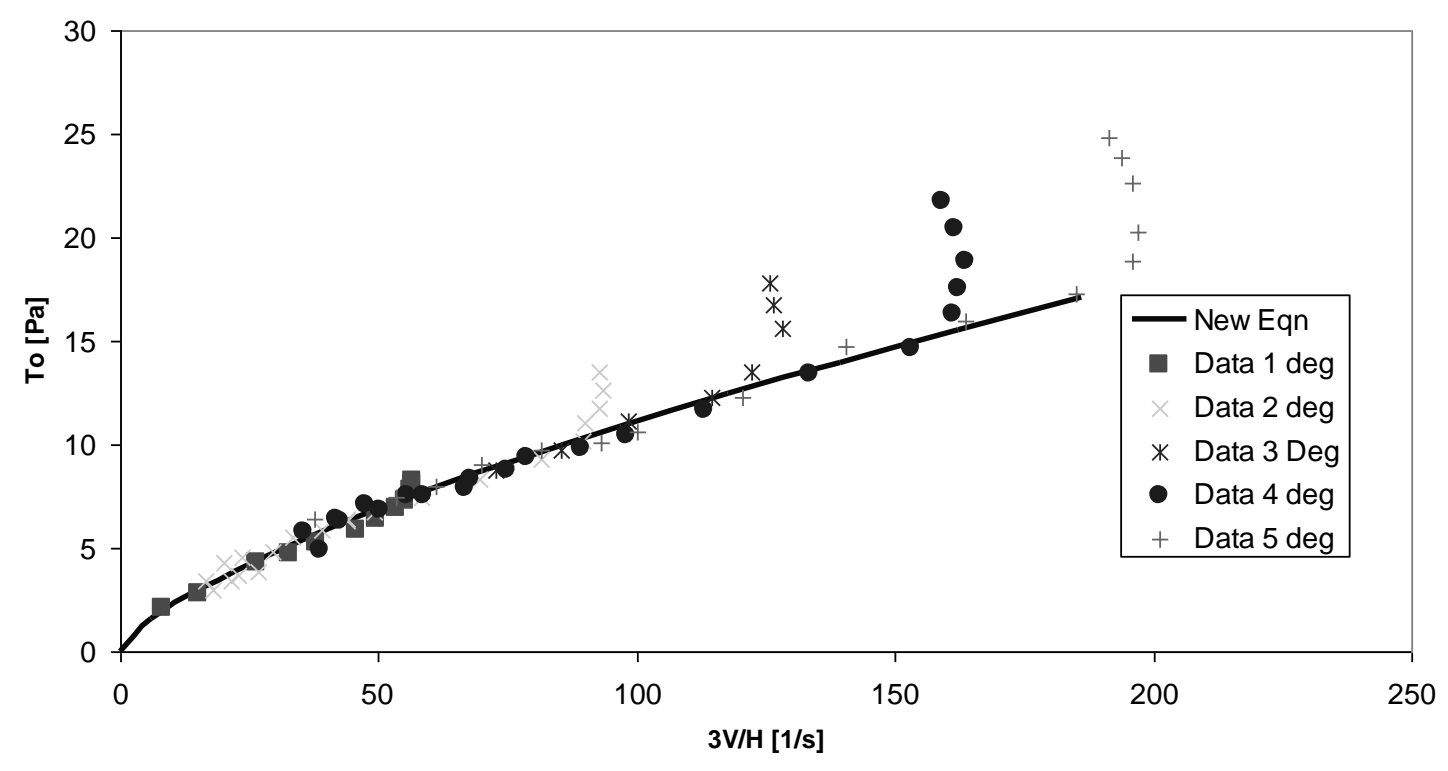

Figure 2 Plot of wall shear stress versus bulk shear rate for a $3.8 \% \mathrm{CMC}$ solution in a $300 \mathrm{~mm}$ rectangular channel

Since the primary objective of this paper is to establish a transitional flow criterion, the first task is to empirically establish the actual transition values.

In order to do this we need to use a normalised adherence function (Slatter, 1999), which indicates the adherence, or otherwise, of data to the new equation (Equation (12)). In this context, we define our normalised adherence function as the wall shear stress ratio $\tau_{0}$ Ratio as:

$$
\tau_{0 \text { Ratio }}=\frac{\tau_{0 \text { Actual }}}{\tau_{0 \text { Laminar }}}
$$

For laminar flow, the value of the normalised adherence function will approach unity for laminar flow, and will rise sharply as the flow becomes turbulent. Thus, by plotting the $\tau_{0}$ Ratio values against $\mathrm{Re}_{4}$, we can establish empirically a transition criterion for sheet flow. Figure 3 shows a plot of $\tau_{0}$ Ratio versus $\mathrm{Re}_{4}$, showing transition criterion for the laminar/turbulent transition. Figure 3 shows that the turbulent data loci from each of the five slopes rises sharply away from unity in the region of $\mathrm{Re}_{4}=700$. On the basis of this empirical evidence, we propose that the laminar/turbulent transition criterion for sheet flow can be established as $\mathrm{Re}_{4}=700$. It should be noted that - as for critical pipe flow - there may well be a zone or range of values 
within which transition can occur. However, a much larger and diverse data base would be required to delimit such a transition zone, and much further work is required.

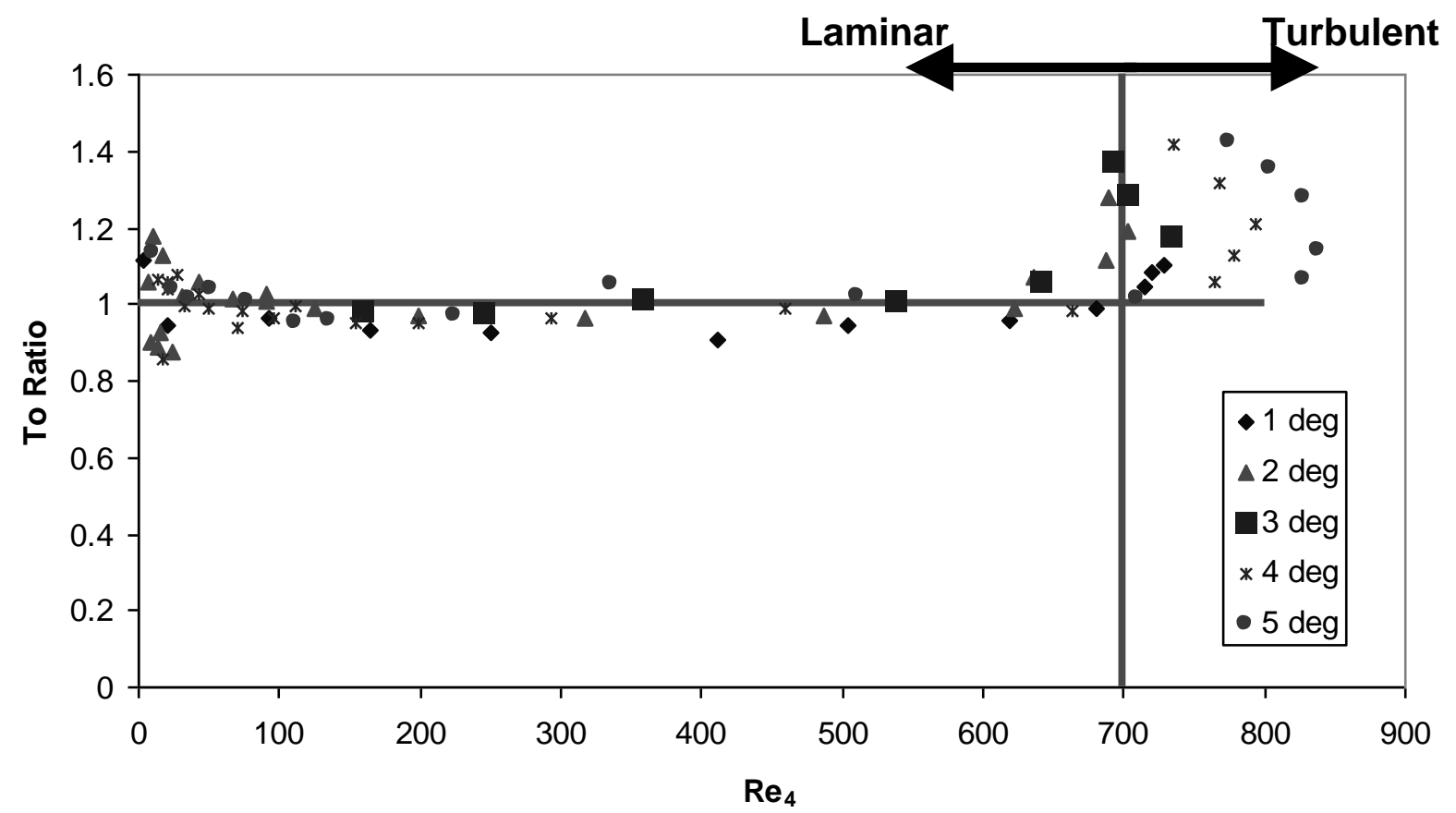

Figure 3 A plot of $\tau_{0}$ Ratio versus $R_{4}$, showing transition criterion

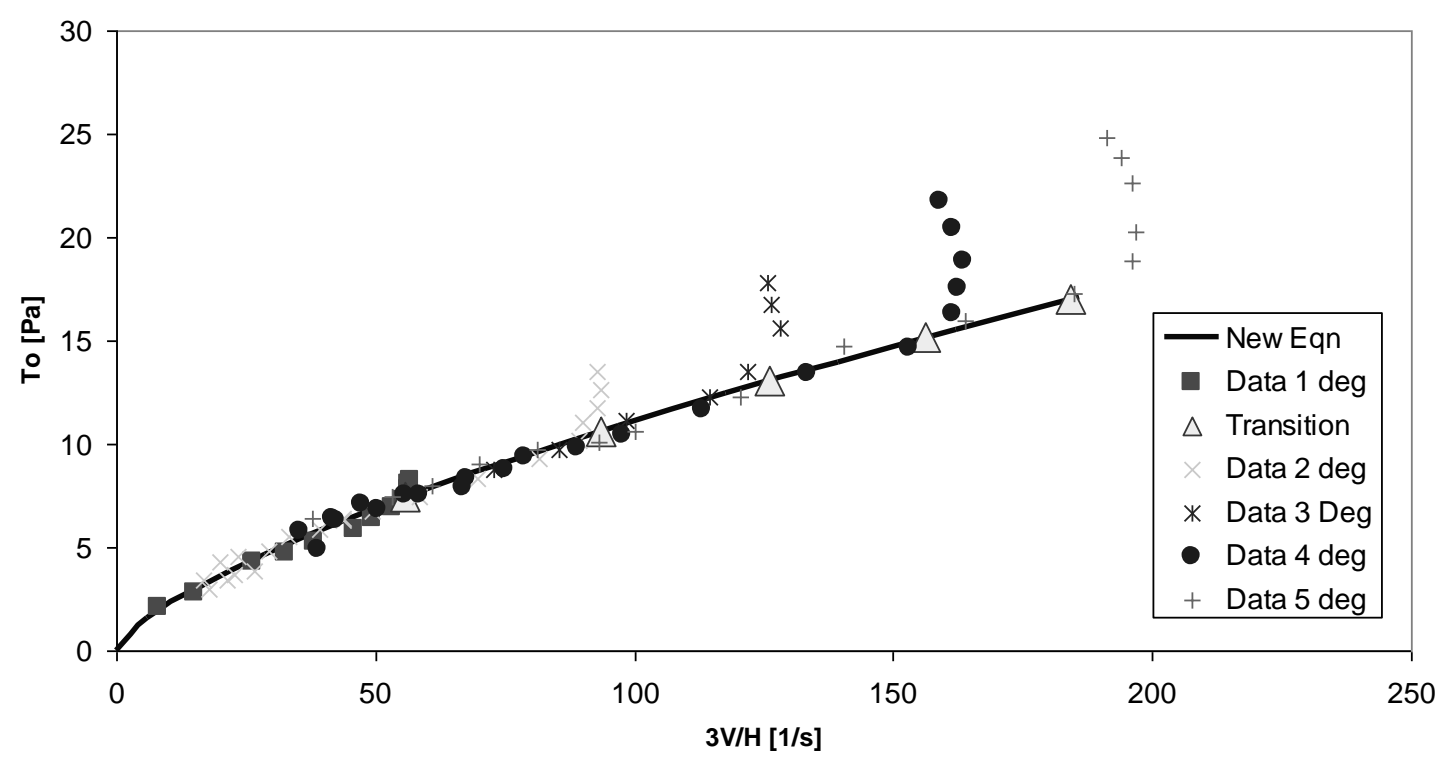

Figure 4 Bulk shear rate diagram showing the calculated transition points at each slope at $\operatorname{Re}_{4}=700$

Figure 4 shows that there is good agreement between the transition predictions obtained using the criterion $\mathrm{Re}_{4}=700$, and the data deviations from the laminar line as turbulence begins.

Further confirmation of the validity of the new criterion can be illustrated by plotting all the data in terms of the new Reynolds Number $\mathrm{Re}_{4}$ and the standard Fanning friction factor $f$. Such a plot is presented in Figure 5 , it shows uniform laminar/transition deviation in the vicinity of $\operatorname{Re}_{4}=700$. 


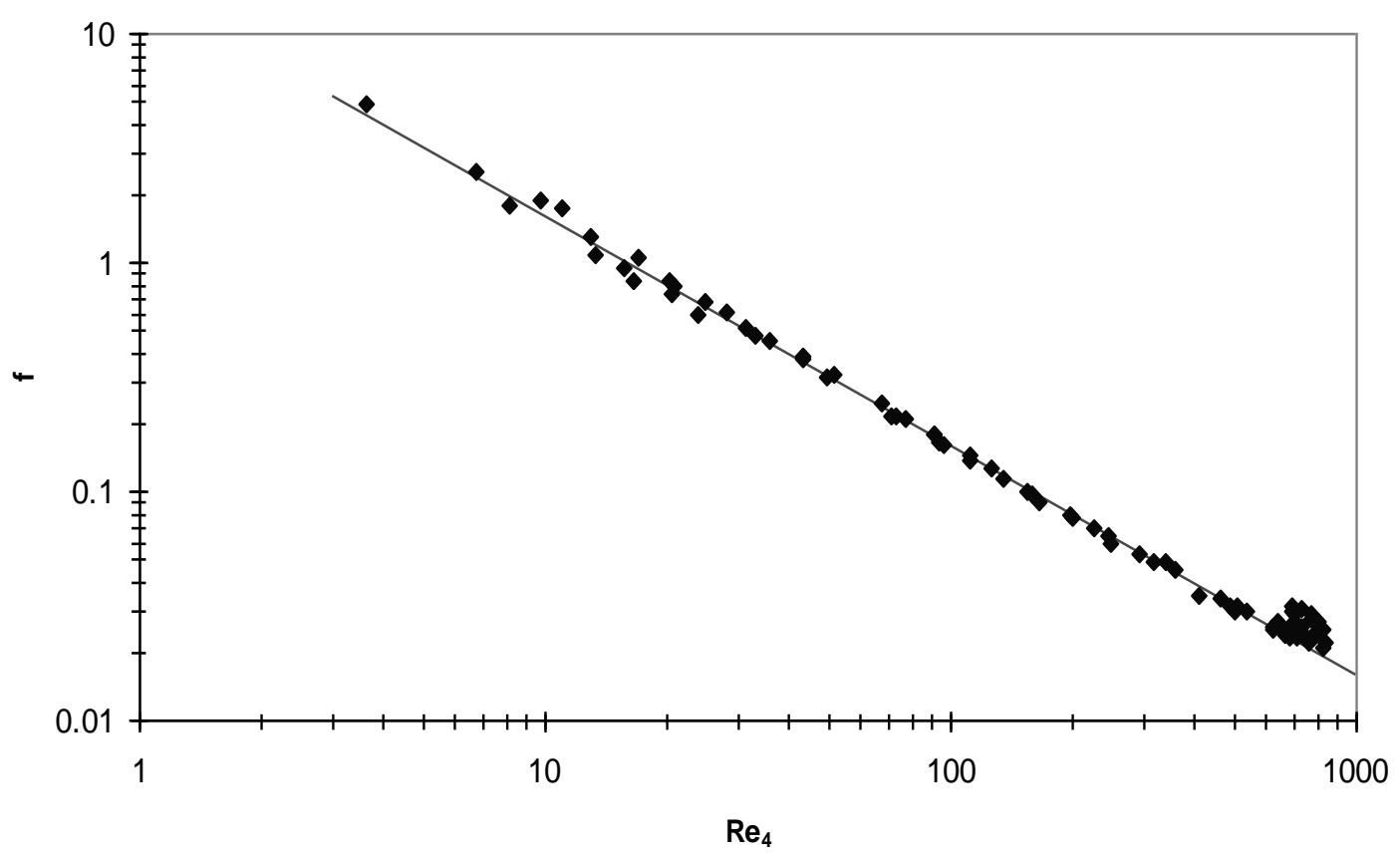

Figure 5 Plot of Fanning friction factor $f$ against the new Reynolds number $\operatorname{Re}_{4}$

\section{Discussion}

One of the principal benefits of the new approach is that laminar flume flow data can now be scaled up directly for engineering design purposes. Alternatively, scale up can be performed via the sheet flow pseudo shear diagram, which can be constructed from the rheology as measured by standard bench-top methods.

Most importantly, the validity of such laminar flow scale up, and the onset of turbulent flow can be predicted from the new transition criterion of $\operatorname{Re}_{4}=700$. The long established dogma - from Newtonian pipe flow - of a transition zone or range of values within which transition occurs, may well apply here. However, much further work over wide ranges of fluid and conduit properties would be required to delimit such a transition zone.

The principal unresolved issues revolve around Froude number or free surface effects. These relate to the phenomena of critical, tranquil or shooting flow, and how these can be related in non-Newtonian open channel flow.

Extension of this approach to visco-plastic fluids may also be possible. However, as for pipe flow, the apparent sheet flow behaviour index will not be constant. A possible avenue to advance this idea is by using the tangent method at the relevant wall shear stress as proposed by Metzner and Reed (1955) which may prove to be viable. However, this remains to be validated.

\section{Conclusions}

A new analytical approach for the sheet flow of a power law fluid has been introduced and further developed. This approach exploits the fact that the bulk shear rate is a unique function of the rheogram and the wall shear stress, and we have shown that this can be used for scale-up and design at any required slope and depth, in laminar flow.

A new criterion for the establishment of the onset of turbulent flow has been developed and validated using experimental data. This approach is analogous to that of Metzner and Reed (1955) for laminar pipe flow.

Unresolved issues have been highlighted. Future work will entail the influence, identification and analysis of the transition to turbulent flow for visco-plastic fluids; the entrance and end effects for sheet flow; identification and analysis of critical flow conditions which are related to surface disturbances and Froude number effects; and the influence, identification and analysis of slip flow effects. 


\section{References}

Chow, V.T. (1959) Open Channel Hydraulics, McGraw-Hill Book Co-Singapore.

Fuentes, R. (2004) Slurry flumes in Chile (Keynote Address), Hydrotransport 16, 16th International Conference on the Hydraulic Transport of Solids in Pipes, Santiago, Chile.

Haldenwang, R. (2003) Flow of non-Newtonian flow in Open Channels, Unpublished D.Tech thesis, Cape Technikon, Cape Town, South Africa.

Haldenwang, R. and Slatter, P.T. (2006) Experimental procedure and database for non-Newtonian open channel flow, Journal of Hydraulic Research, Vol. 44, Issue 2, pp. 283-287.

Haldenwang, R., Slatter, P.T. and Chhabra, R.P. (2002) Laminar and Transitional flow in Open Channels for nonNewtonian Fluids, in Proceedings 15th International Conference on Hydrotransport, Banff, Canada, pp. 755-768.

Metzner, A.B. and Reed, J.C. (1955) Flow of non-Newtonian fluids - correlation of the laminar, transition and turbulent flow regions, AIChE Journal, Vol. 1, No. 4.

Slatter, P.T. (1999) A new friction factor for yield stress fluids, British Hydromechanics Research Group 14th International Conference on Slurry Handling and Pipeline Transport Hydrotransport 14, Maastricht, September, pp. 255-265.

Slatter, P.T., Haldenwang, R. and Chhabra, R.P. (2010) The Sheet Flow Viscometer, Hydrotransport 18, in Proceedings 18th International Conference on the Hydraulic Transport of Solids, BHR Group, Rio de Janeiro, 22-24 September. 